\title{
Vibration Resistance Structure Optimization Design on Special Vehicle of Fuel Tank
}

\author{
Zhang Hongwang ${ }^{1, \mathrm{a}}$, Liu Nan $^{12, \mathrm{~b}}$, Liu Jinli $^{1, \mathrm{c}}$,Shao Weiguang ${ }^{1, \mathrm{~d}}$, Xie Tao $^{1, \mathrm{e}}$ \\ 1 POL Research Institute of Beijing, Beijing, 102300, China \\ 2 Department of Precision Instrument and Mechanisms, TsingHua University, Beijing, 100084, China

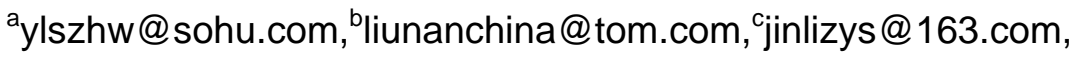 \\ dshaowg@163.com, ${ }^{\mathrm{e}}$ tony1981001@126.com
}

Keywords: fuel tank, vibration resistance structure, stiffness, strength

\begin{abstract}
In order to improve the vibration resistance performance of the special vehicle's fuel tank, enhance the safety, avoid the structure occurring fatigue damage in the life period, reduce the weight of fuel tank, comprehensive consideration of the beauty of the structure and the economy of the manufacturing process, the new fuel tank of rectangular thin-walled container is been designed based on the sheet steel welding structure forms, and it meets the actual working condition and has very good vibration resistance performance through optimization structure and comparing the processes. Finally, it can completely meet the needs of engineering application. Meanwhile, the optimized calculation and research analysis provide credible theory and experience for engineering application.
\end{abstract}

\section{Introduction}

The structure of the traditional fuel tank on the vehicle is always a kind of the round head welded the cylinder body structure since the force is simply and uniformly distributed. But the fuel tank for special vehicle is working in complex condition, as well as the space requirements are also extremely restriction. Based on the space, the fuel tank often is designed as the rectangular section tank or irregular shape, in order to meet vibration requirements about the structure of special vehicle's fuel tank. [1]

The fuel tank is usually made from thin steel plate welded together. Traditional method uses inner skeleton support the steel plates welding structure in order to improve the stiffness of the tank, obviously this structure form in some places need skinned spot welding inevitably to fix and connect the different parts together.

But there are a lot of security problems about welding joint of fuel tank. A welded joint is the site where the whole structure is most likely to occurring fatigue failure normally, especially in frequent vibration process when the special vehicle is in the working condition. The welding crack leads to tank leakage occurring frequently, and resulting in security incidents.

\section{Welded joint design}

The structure of fuel tank is shown in fig1.It is made from skeleton, bottom plate, side plate and upper plate. After the stress analysis by finite element software, the maximum stress of the structure appear in the junction of bottom plate and side plate(fig2),and the local stress contour is in the fig3. 


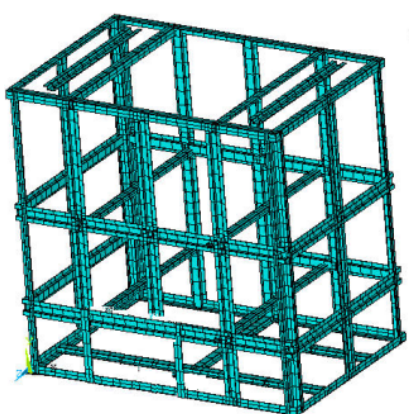

fig1 reinforcement structure

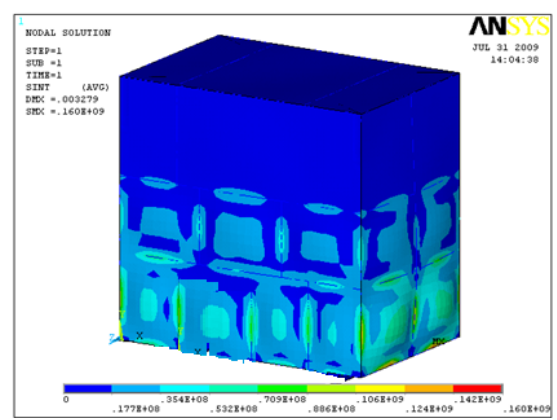

fig2 computer stress analysis

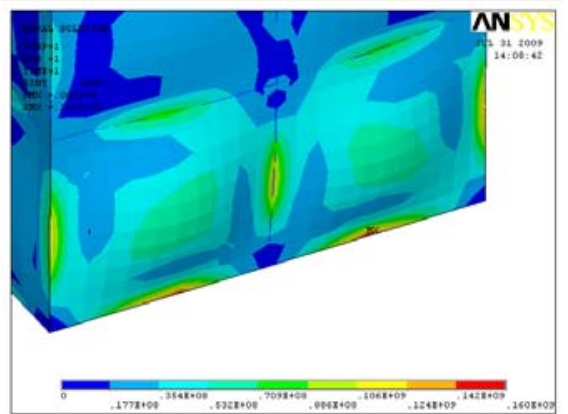

fig3 local stress contour

From the analysis chart, the welding joint between bottom plate and side plate is very important for the entire structure. Considering the four structure as follows.(fig4-7) the bottom and side panels are connected together .

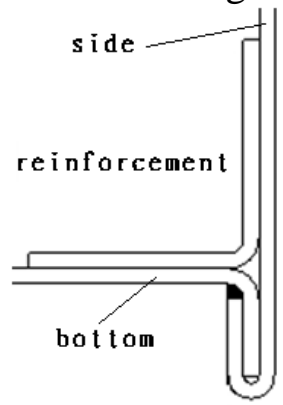

fig4 structure1

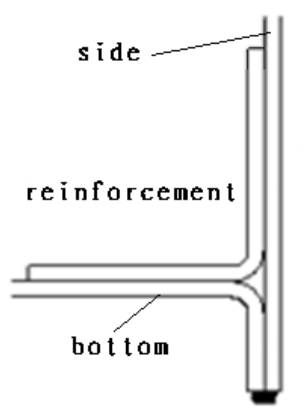

fig5 structure2

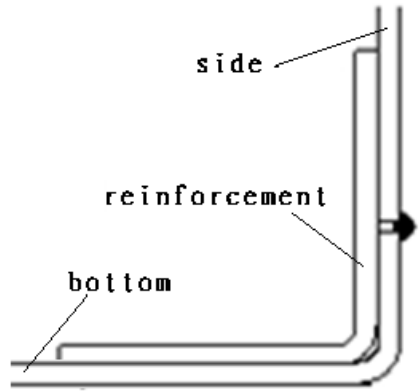

fig6 structure3

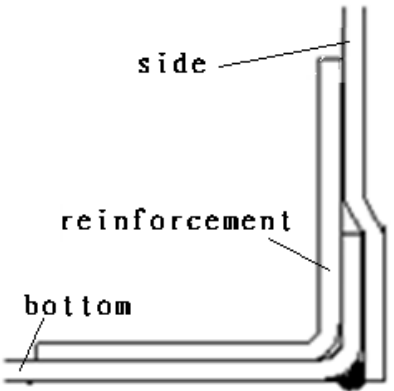

fig7 structure4

After finite element simplified analysis and the practical engineering experience, the structure 1 is eventually adopted as the process of welding connection. Through vibration test, this welding method is conducive to the production and processing, and achieves the design requirements about strength and stiffness. Since batch production, has not received welding parts fatigue damage occurred.
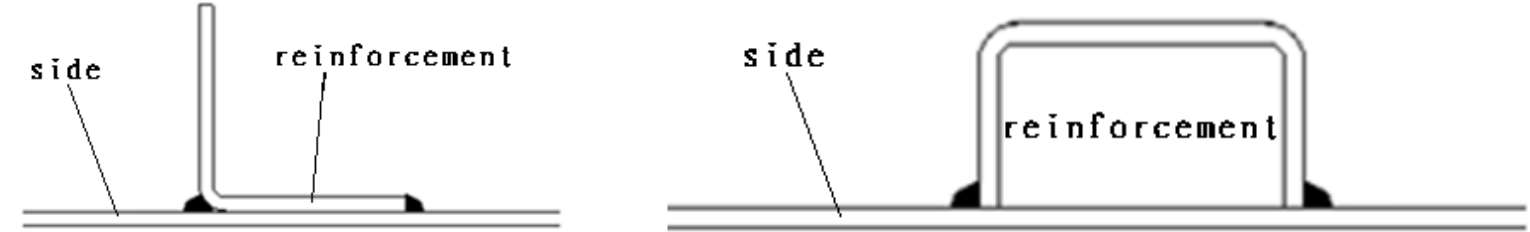

fig8 side panel welded angle steel rib

fig9 side panel welded Channel steel rib

Traditional fuel tank adopts the rib support the plate of the body. The fig8-9 are the two way, side panel welded angle steel rib and side panel welded channel steel rib .But in order to increasing the volume of fuel tank and ensuring the stiffness, increasing the number of internal ribs in the fuel tank is not a good way.

New design fuel tank structure should have the characteristics about the simple structure and process technology, little deformation of steel sheet under pressure and well stiffness and strength. So the design about large volume fuel tank not only considers the welding technology to strengthen the weld structure strength, but also improves static strength and dynamic fatigue design by using stamping technology to produce the side panels of fuel tank. It eliminates welding spots fix for internal reinforced ,and is helpful to improve the vibration resistance performance of the special vehicle’s fuel tank.[2]

\section{Tank topography optimization}

The entirety fuel tank is the a design object, It solves the following structural optimization problem: 
$\min f(x)=f\left(x_{1}, x_{2}, \ldots, x_{n}\right)$

$\begin{array}{ll}\text { Subject to: } & g_{j}(\mathrm{x}) \leq 0 \quad j=1, \ldots, m \\ & x_{l} \leq x_{l} \leq x_{l}^{u} \quad i=1_{1}, \ldots, n\end{array}$

The objective function $\mathrm{f}(\mathrm{x})$ and the functions $\mathrm{g}(\mathrm{x})$ in the constraint function are structural responses obtained from a finite element analysis. A constraint is considered active if it is satisfied exactly ( $\mathrm{g}=0$ ); it is considered inactive if $\mathrm{g}<0$; it is considered violated if $\mathrm{g}>0$.

The selection of the vector of design variables $x$ depends on the type of optimization being performed. In topology optimization, the design variables are element densities

In size optimization, the design variables are properties of structural elements. In topography and shape (including free-shape) optimization, the design variables are the factors in a linear combination of shape perturbations.[3]
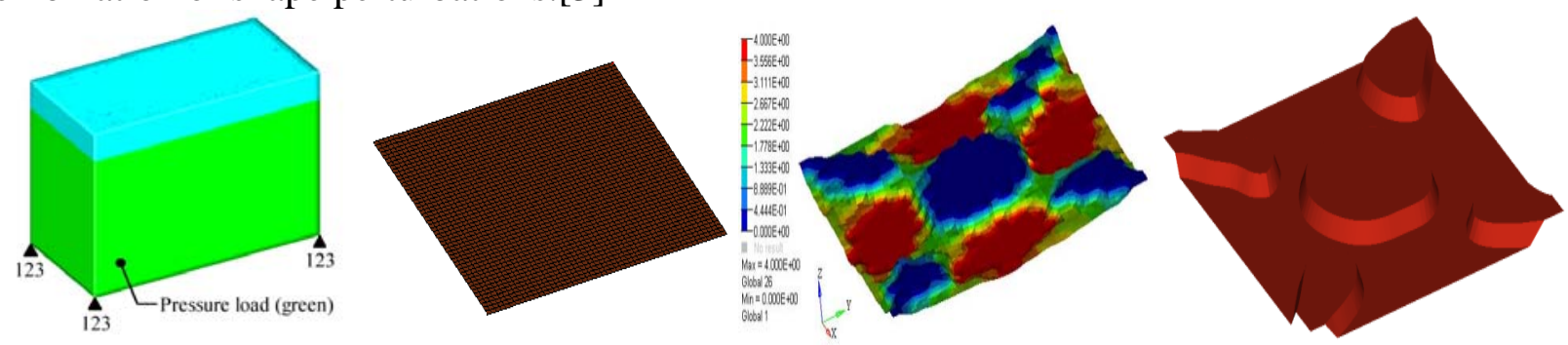

fig10 model fig11 simplified model fig12 topography optimization fig13 auto-recognition result

Setup model. As the design object, a rectangular, thin-walled container using for storing fluid is set up and the finite element model of the design space with loads and constraints is described as the fig10. According to the optimization design method, the objective, constraints and the design variables is stated as following table1.

Table 1 the optimization problem for fuel tank

\begin{tabular}{|c|l|}
\hline Objective: & $\begin{array}{l}\text { Minimize the outward bulging of the sides of the container caused by the } \\
\text { pressure of its contents }\end{array}$ \\
\hline Constraints: & $\begin{array}{l}\text { Displacement in all directions is at the four lower corners, but is free to } \\
\text { rotate about those constraints. The loading is a distributed pressure } \\
\text { through the area shown in green. The pressure is higher at the bottom of } \\
\text { the vessel. }\end{array}$ \\
\hline $\begin{array}{c}\text { Design } \\
\text { variables: }\end{array}$ & $\begin{array}{l}\text { Shape variables generated automatically on the designable space aligned } \\
\text { with the elements normal. }\end{array}$ \\
\hline
\end{tabular}

Model simplification. The fuel tank is a symmetrical structure as known. In order to simplifying the research problem and the optimization model, the side panel as the simplified model is studied as fig11 shown. So the optimization problem is transformed into topography optimization of a plate under pressure. A finite element model of the design space with loads and constraints applied is shown in the fig11. It is assumed that the part is to be formed using a stamping process. The objective is to minimize the displacement of the node where the force is applied in the positive z-direction. Only the shape of the plate can be changed to achieve the objective, not the thickness.

Then, the constraints, load, material properties, and subcase of the model are already defined. Topography design variables and optimization parameters are defined and the software is used to determine the optimal reinforcement patterns.

Result analysis. The results are viewed as animations of the contours of shape changes of the design space as fig12. After the automatic recognition of bead results, the optimization result is shown as the fig13. Finally, considering the process needs, the actual beauty and the commonality of the product, based on the above topography optimization result, by using of the grouping patterns is shown, based on the shape changes suggested by software, a possible pattern is chosen for ease of 
manufacturing, the new topography optimization design is got after adding pattern grouping constraint. The fig14 is the contour plot showing the reinforcement pattern with pattern grouping constraint. Finally, the optimization result is the automatic recognition of bead results as the fig15.

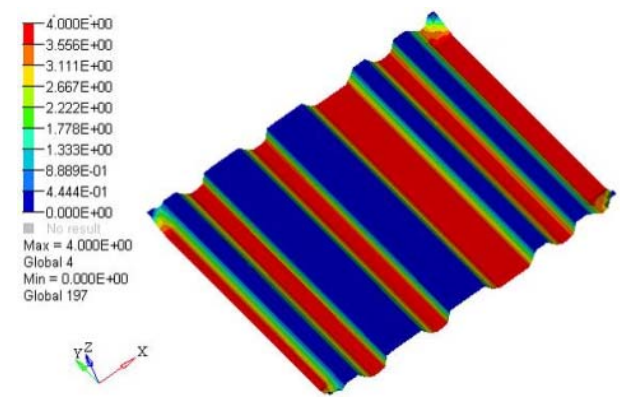

fig14 add pattern grouping constraint fig15 auto-recognition results

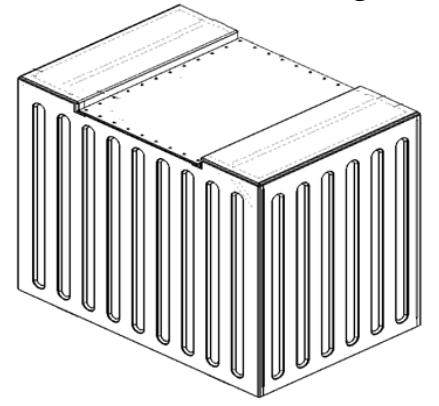

fig16 final structure

Ultimately, consideration economy of processing technology and mold design and processing, manufacturing, final structure of fuel tank is shown as the fig 16.Throught the vibration test and batch product application, fuel tank stiffness and strength of the specialty vehicle, meet the design requirements, and the vibration resistance performance meet the actual working condition requirements.

\section{Conclusion}

Comparing and analyzing the series of welded joint structure about fatigue properties, the fuel tank adapts reliable weld process to improve the structure of vibration resistance capability, increasing the reliability and prolonging the life of the fuel tank in the special vehicle. Through vibration test, this welding method is conducive to the production and processing, and achieves the design requirements about strength and stiffness.

Based on computer application in design and manufacturing, the finite element analysis method and optimization design theory, a rectangular, thin-walled fuel tank is designed. After topography optimization and adding pattern grouping constraint, consideration economy of processing technology and mold design and processing, manufacturing, final optimization structure of fuel tank is produced and manufactured.

Using finite element analysis and the optimization software for the design, it can accelerating product design speedily and reliability and reducing product development cycle and cost. In addition, the research lays the foundation for the development of similar products.

\section{Reference}

[1] Chen Jushu,TU Minglin,Chen Junli, Vibration Resistance Performance of the Special Vehicle's Fuel Tank, Pipeline Technique and Equipment, 2011.1, (In Chinese)

[2] Nan Liu, Jun Zhao, Modal Analysis and Optimization Design on a Vehicle-load

Rectangle Liquid Containers under Fluid-structure Coupling, Applied Mechanics and Materials Vol. 120 (2012) pp 444-447

[3] Nan Liu, Hongwang Zhang, Strength and Modal Analysis of Thin-shell-structured Vessel with Rib Reinforcement, Advanced Materials Research Vols. 472-475 (2012) pp 2722-2725 